\title{
Pensamento matemático-computacional
}

\section{Mathematical-computational thinking}

\author{
1 Christian James de Castro Bussmann christian@uenp.edu.br \\ 2 Angela Marta Pereira das Dores Savioli
}

$1 \quad$ Universidade Estadual do Norte do Paraná

2 Universidade Estadual de Londrina

\section{Resumo}

Este artigo apresenta os resultados de uma pesquisa que teve como objetivo construir uma teorização, tendo como base os processos do Pensamento Matemático Avançado apresentados por Dreyfus (2002) e as concepções do Pensamento Computacional. A metodologia utilizada foi a da pesquisa especulativa, que são declarações teóricas de outras declarações. Concluiu-se que o Pensamento Matemático-Computacional é uma teorização e algumas de suas características, como relação entre conceito e simbologia, representações concretas, inteirações e observação de padrões, ações que envolvem padrões, reflexões, diálogo e arguição, conexão entre os assuntos da disciplina, experienciação da evolução do pensamento científico, construção da notação e sistema de representações. Além disso, a atuação docente em sala de aula pode contribuir para evidenciá-la, ou seja, o Pensamento Matemático-Computacional estabelece a relação docente - aluno e aluno - docente.

\section{Palavras-Chave}

Educação matemática. Pensamento matemático. Pensamento computacional. Pensamento matemático-computacional.

\section{Abstract}

This article presents the results of a research that aimed to build a theorization based on the Advanced Mathematical Thinking processes presented by Dreyfus (2002) and the concepts of Computational Thinking. The methodology used was that of speculative research, which are theoretical statements of other statements. It was concluded that MathematicalComputational Thinking is a theorization and some of its characteristics, such as the relationship between concept and symbology, concrete representations, interactions and observation of patterns, are actions involving patterns, reflections, dialogue and argument, connection among the subjects of the discipline, experience of the evolution of scientific thought, construction of notation and system of representations. In addition, the teaching performance in the classroom can contribute to highlighting it, that is, MathematicalComputational Thinking establishes the teacher student and student - teacher relationship.

\section{Keywords}

Mathematical education. Mathematical thinking. Computational thinking. Mathematical-computational thinking.

\section{Como você deve citar?}

BUSSMANN, Christian James de Castro; SAVIOLI, Angela Marta Pereira das Dores. Pensamento matemático-computacional. Cadernos UniFOA, Volta Redonda, n. 42, p. 91-100, abril, 2020. 


\title{
1 INTRODUÇÃO
}

Este artigo apresenta o resultado de uma pesquisa que culminou em uma teorização denominada Pensamento Matemático-Computacional e, para tal, utilizou-se os processos envolvidos no Pensamento Matemático Avançado (Dreyfus 2002) e nas concepções do Pensamento Computacional.

Buscou-se unir as discussões apresentadas por Dreyfus (2002) sobre os processos que envolvem o Pensamento Matemático Avançado e os pesquisadores que discutem o Pensamento Computacional, construindo, assim, uma teorização denominada Pensamento Matemático-Computacional.

Para tal, utilizou-se como perspectiva metodológica as concepções da Pesquisa Especulativa, que são declarações teóricas surgidas de outras declarações teóricas. $O$ resultado foi o desenvolvimento de processos simbólicos, mentais e de abstração que foram construídos por meio de interpretação. Dessa forma, este artigo tem como objetivo apresentar uma teorização, tendo como base os processos do Pensamento Matemático Avançado apresentados por Dreyfus (2002) e as concepções do Pensamento Computacional.

\section{FUNDAMENTOS TEÓRICOS E METODOLÓGICOS}

Antes de iniciar as discussões teóricas a respeito da Construção Simbólica do Pensamento Matemático-Computacional, entende-se que seria interessante apresentar algumas das concepções que envolvem o ensino da Matemática, tanto em nível básico, quanto em nível superior.

Dessa forma, pode-se afirmar que a prática do professor de Matemática é recheada de representações e, para realizá-las, os símbolos são primordiais.

De acordo com Olson e Campbell (1994), em muitos casos, os símbolos servem para representar o conhecimento, no entanto, eles também argumentam que existe um perigo nessa relação entre o símbolo e essa representação.

Para Olson e Campell (1994, p. 9) os estudantes

\begin{abstract}
[...] começam a enxergar a possibilidade do simbolismo, isto é, representar algo por meio de outra. Nesse estágio, ela aprende que algo pode simbolizar ou representar outra coisa ou uma classe de coisas. No próximo estágio, a criança entende que o símbolo refere-se, e pode ser usado para representar um objeto ou um evento específico. $\mathrm{Na}$ terceira fase, eles começam a entender que a representação de um objeto pode ser verdadeira ou falsa. É somente na terceira fase que ele adquire essa meta-representação de um conceito ser verdadeiro ou falso, que afirmam ser um conceito mais amadurecido.
\end{abstract}

As mesmas ideias são compartilhadas com Dreyfus (2002, p. 30), pois esse autor também acredita que "[...] haver algum significado associado a uma noção antes de um símbolo para que a notação possa ser de alguma utilidade".

Harel e Kaput (2002) fazem uma discussão sobre a importância da simbologia e seu papel para a construção da Matemática. Para eles, o entendimento da simbologia passa por duas fases: a primeira fase deve discutir a construção das entidades conceituais e sua relação com a notação matemática; na segunda fase, discute-se o papel das notações no Pensamento Matemático e sua contribuição para o crescimento vertical da Matemática, que se configura por um amadurecimento matemático.

Cada uma dessas fases passa por alguns estágios. Na primeira fase das entidades conceituais, tem-se três estágios: 
- quando o conceito é constituído de vários elementos. Inicialmente, separa-se em etapas para melhor compreender assuntos complexos;

- em um segundo momento, inicia-se a inserção dos operadores e como são utilizados dentro dessa entidade conceitual e;

- o terceiro estágio permite ao estudante utilizar uma estrutura apropriada para a resolução de um problema.

A segunda fase, que discute a notação matemática, também passa por três estágios:

- o primeiro discute o papel da notação na formação das entidades conceituais;

- o segundo apresenta uma discussão entre os diferentes tipos de notação e sua utilização na representação estrutural de um conceito e;

- no último estágio, a notação como substituta dos conceitos.

Ainda de acordo com Harel e Kaput (2002, p. 94), um dos problemas para o entendimento da simbologia matemática se encontra na própria maneira como atuamos em sala de aula, pois enquanto os inventores das notações

\footnotetext{
[...] as criaram para expressar e talvez elaborar suas próprias concepções pré-existentes, nas escolas frequentemente começamos em ordem inversa, concentrando-nos na manipulação de notações, [...] Os estudantes deveriam ter a oportunidade de construir suas próprias expressões de notação das suas ideias, que podem ser guiadas na direção dos padrões. Desta forma, constrói-se notações e concepções simultaneamente, em vez de construir um e depois o outro e, em seguida, tentar ligar os dois.
}

Mesmo com toda essa discussão sobre como ensinar os símbolos e utilizá-los na aprendizagem, Dreyfus (2002) ainda argumenta que outros tipos de Representações (geométrica, tabular, entre outras) podem auxiliar para o pensar em matemática. Nessa perspectiva, a representação mental pode contribuir para o ensino da Matemática.

Dreyfus (2002) afirma que a representação mental são esquemas internos ou quadros que são utilizados para interação com o mundo externo e que cada sujeito tem sua própria representação. Por exemplo, o professor, ao abordar um assunto, pode ocorrer de surgir representações mentais divergentes, ou seja, enquanto para alguns as derivadas estão relacionadas à "regra do tombo" $\left(f^{\prime}(x)=n x^{n-1}\right)$, para outros está associada ao estudo da reta tangente.

Logo, pode-se afirmar que, em uma sala de aula, podem ocorrer, pelo menos, dois tipos de representações mentais, a do professor e a do estudante. Dreyfus (2002) entende que essa distinção é significativa, pois enquanto o discente pensa em como resolver, o docente pode pensar como um objeto possui uma determinada complexidade, com definições e teoremas associados.

Vinner (2002) nos fornece uma ideia de como se dá a formação de um conceito por meio da concepção de conceito imagem. Segundo o autor (2002), o conceito imagem é algo não verbal que está associado a um nome, podendo ser uma representação visual ou uma compilação de impressões e experiências que podem ser traduzidas em formas verbais.

Tanto Vinner (2002) quanto Dreyfus (2002) argumentam que uma representação é rica quando contém uma quantidade de aspectos ligados ao conceito. Dessa maneira, fazem-se duas inferências: 
a primeira é que possuir uma representação visual é ter uma quantidade de impressões e experiências sobre o conteúdo; a segunda é que as representações mentais dos estudantes se desenvolvem com base nas representações concretas que são evidenciadas pelo professor.

De acordo com Dreyfus uma representação é rica quando possui uma quantidade de aspectos ligados ao conceito. Dessa maneira, possuir uma representação visual é ter uma boa impressão e experiência sobre o conteúdo.

Apresentam-se, agora, alguns aspectos do Pensamento Computacional que contribuíram para o desenvolvimento dos processos de Construção Simbólica e Mental do Pensamento Matemático-Computacional.

Wing (2006) aborda, pela primeira vez, o termo Pensamento Computacional e defende que ele é útil na resolução de problemas, no desenvolvimento de sistemas e na compreensão do comportamento humano e que, de acordo com a autora, são elementos importantes para Ciência da Computação.

Posteriormente, Wing (2010) acrescentou novos elementos a essa definição, argumentando que o Pensamento Computacional também tem utilidade em outras áreas do conhecimento. Dessa maneira, 0 Pensamento Computacional deixou de ser exclusivo de profissionais da área de computação e passou a ser uma linha de pesquisa que pode contribuir para outras áreas do conhecimento.

Em 2010, na cidade de Boston, foram realizados dois workshops para discutir sobre o Pensamento Computacional em que foram apontadas algumas vertentes. Entre elas, destacam-se: como uma linguagem e a importância da programação; como automação de abstrações; como uma ferramenta cognitiva e um contexto sem o computador.

Para este trabalho, apresentam-se as vertentes da linguagem e importância da programação e como automação de abstrações, já que elas contribuíram no desenvolvimento da construção simbólica e mental.

Neils Bohr (2010), um dos participantes do Comitê, alega que o Pensamento Computacional deve ser encarado como uma linguagem que as pessoas utilizam para entender diversas situações, bem como problemas complexos, e como trabalhar com eles.

Outros pesquisadores, como Roy Pea (2010) e Ursula Wolz (2010), vão além: para se aprender essa linguagem, é necessário entender de programação. Além disso, esse é um dos elementos principais para uma compreensão do Pensamento Computacional.

Resnick (2010, p. 14) argumenta que o Pensamento Computacional é mais do que somente programar. Para ele, a programação é um "[...] meio de expressão e um ponto de entrada para o desenvolvimento de novas formas de pensar".

Esse entendimento do Pensamento Computacional como uma linguagem e que a forma de expressá-lo se dá por meio da programação permitiu que as pessoas construíssem e compreendessem o que antes não era possível.

Outro participante do Comitê, Alan Kay (2010), não tem a mesma compreensão. Mesmo reconhecendo o Pensamento Computacional como sendo uma linguagem que permite descrever aspectos universais, para ele, nem todos os seres humanos conseguirão entendê-la. De acordo com Kay (2010, p. 16), 
[...] todos os seres humanos têm uma capacidade inata de linguagem verbal, mas que o mesmo não pode ser dito para a Linguagem escrita, a ciência e a matemática dedutiva, porque estas não são encontradas em todas as culturas ou sociedades. Este ponto sugere que, seja qual for o Pensamento Computacional - como idioma, os seres humanos não aprenderão o Pensamento Computacional do mesmo modo que aprendem a falar. Por outro lado, ele também observou que um aspecto poderoso do Pensamento Computacional implica na capacidade de criar uma Linguagem bem adaptada a um propósito pessoalmente relevante - e, de fato, essa habilidade poderia ser ensinada aos estudantes.

Nessa perspectiva, a programação é um elo que pode contribuir para o desenvolvimento do Pensamento Computacional. No entanto, apresenta dificuldades, como acontece com o Pensamento Matemático e a Linguagem Matemática. Contudo é de comum acordo que ambos contribuem de forma significativa para o entendimento de diversas tarefas e podem ser trabalhados com os estudantes, aprimorando seus conceitos e, além disso, desenvolvendo novas habilidades.

Ainda, o Pensamento Computacional pode possibilitar novas descobertas, permitindo às pessoas acessarem e organizarem informações de maneira nunca feita antes, acessando informações digitais ou como Fox (2010, p. 16), membro do Comitê, argumenta, "[...] modelando e representando algo que fazemos de modo exclusivo e com diversas maneiras de explicar e enriquecer", ou seja, o Pensamento Computacional permite que as pessoas possam fazer suas descobertas, que cada um possa construir novos elementos e uma forma diferenciada de explicá-los.

Outros participantes do Workshop defendem a ideia de que o Pensamento Computacional pode ser compreendido como um processo de criação, gerenciamento de abstrações e estudo de relações entre leis de abstração.

Nessa perspectiva, o Committee for the Workshop on Computacional Thinking (2010) entende que Pensamento Computacional

[...] é uma ferramenta para explicar e representar a complexidade por meio da automação. Embora a matemática e a física também estejam centralmente preocupadas com o uso da abstração para gerenciar e controlar a complexidade, os métodos computacionais adicionam outra dimensão ao controle da complexidade - a da automação (COMMITTEE FOR THE WORKSHOPS ON COMPUTATIONAL THINKING; NATIONAL RESEARCH COUNCIL, p. $16-17,2010)$.

Segundo Peter Lee (2010), o Pensamento Computacional vai além de ser somente uma ferramenta, auxiliando na aprendizagem e utilizando conceitos de automação e resolução de problemas.

Além disso, diSessa (2010) e Astrachan (2010) entendem que o Pensamento Computacional colabora na explicação de determinados assuntos para a comunidade de modo geral. Para esses pesquisadores, uma situação é falar para profissionais da área de tecnologia e outra é falar aos demais profissionais.

Khan $(2010,2010$, p. 17) argumenta que o Pensamento Computacional pode ajudar na concretização, na "[...] criação de algo concreto e tangível - de assuntos que são predominantemente abstratos". O mesmo autor aponta, como exemplo, a questão de jogos computacionais, que são virtuais, mas apresentam uma característica concreta.

Para Wielinsky (2010), a importância dessa discussão reside na possibilidade de os estudantes interagirem e, além disso, observarem padrões existentes, resultando em ações concretas no sujeito.

Do ponto de vista metodológico, é um trabalho que apresenta características qualitativas e teóricas, pois o Pensamento Matemático-Computacional é uma teorização científica. Não seria uma teoria em si, pois Godde e Hatt (1979, p. 12) afirmam que, para os cientistas, a "[...] teoria se refere a relação entre fatos, ou à ordenação significativa desses fatos". 
De fato, esta pesquisa se deu buscando relacionar elementos que estão envolvidos nos processos do Pensamento Matemático Avançado descritos por Dreyfus (2002) e nos trabalhos realizados em um Workshop (2010) sobre o Pensamento Computacional, surgindo o Pensamento Matemático-Computacional. Opta-se pela estrutura da pesquisa especulativa em educação.

De acordo com Martineau, Simard e Gauthier (2001), a pesquisa teórica especulativa em educação consiste em três eixos: interpretar, discutir e contar. Assim, um trabalho que assume essa metodologia produz declarações teóricas de outras declarações teóricas.

Segundo os autores, um trabalho especulativo não parte de uma situação empírica, mas visa ser interpretado, avaliando os pontos positivos e negativos, fazendo escolhas e as apoiando por meio de um argumento.

Outra característica da pesquisa especulativa está na construção do "real". Para Plot (1986, p. 14), "[...] o autor de uma tese em Ciências Humanas deve, portanto, constituir por escrito seu "real" para que pareça tão convincente quanto possível".

Outra caracterização da pesquisa especulativa se baseia nas discussões de Judith Schangler (1983) sobre o trabalho intelectual. Para a autora, a pesquisa especulativa também é dividida em três etapas: 1) cognitiva, pois aborda um conhecimento; 2) discursiva, porque necessita ser comunicada e; 3) descrita em um trabalho.

Dessa maneira, uma pesquisa especulativa não necessariamente parte de um trabalho empírico, ou seja, não ocorre por meio de um processo de observação de um determinado experimento e a descrição desses resultados, mas interpretando, realizando escolhas, defendendo-as em um processo argumentativo tanto na construção do "real" (PLOT, 1986) como no desenvolvimento do trabalho intelectual (SCHANGLER, 1983).

Nessa perspectiva, quando os autores afirmam que um dos pilares da pesquisa especulativa é a construção de declarações teóricas tendo como base outras, temos que o Pensamento MatemáticoComputacional foi construído por meio dessa premissa, visto que foram estudados os processos que Dreyfus apresenta para o Pensamento Matemático Avançado, bem como o resultado das discussões ocorridas nos Workshops que debateram a respeito do escopo e das ações pedagógicas do Pensamento Computacional.

\section{RESULTADOS}

Ao iniciar discussões dos resultados encontrados nesta pesquisa, deve-se esclarecer que está se buscando uma comunicação entre o Pensamento Matemático e o Pensamento Computacional. Nessas leituras, detectam-se alguns elementos em comum e outros um tanto divergentes, com destaque para a abstração.

Tomou-se, como linha mestra, as concepções apresentadas por Dreyfus (2002), mas, à medida que se foi construindo os processos que envolvem o Pensamento Matemático-Computacional, observou-se que houve a necessidade de inserir entendimentos sobre cada uma das fases envolvidas, sejam elas de construção simbólica ou construção mental.

Inicia-se essa discussão a partir de algumas características das representações apresentadas por Dreyfus (2002), começando pela representação simbólica. 0 modo como são apresentados os sím- 
bolos, aos estudantes, na maioria das vezes, é realizado de maneira muito próxima ao que aparece em livros didáticos, com a introdução de letras e somente com o professor dando significado aos símbolos.

No entanto, Dreyfus, tomando como argumentação teórica os pesquisadores Olson, Campebell (1994) e Harel e Kaput (2002), entende que o processo de inserção dos símbolos pode ser suavizado, fazendo com que, inicialmente, os estudantes observem a necessidade de construir alguma simbolização de um determinado objeto, ou seja, a ação do professor não seria impor o símbolo, mas sim discutir sobre o assunto e fazer algo sem a utilização da simbologia usual que a Matemática possui. Assim, o próprio estudante vai sentir a necessidade de construir seus símbolos para representar um determinado objeto matemático.

Nessa perspectiva, pode-se fazer um trabalho em conjunto com o Pensamento Computacional, em especial, quando se discute a importância da Linguagem e o papel das entidades conceituais (HAREL; KAPUT, 2002), e qual a sua relação com a notação Matemática.

Por outro lado, o Pensamento Computacional apresenta algumas características que estão associadas à discussão da linguagem e sua importância para a programação, que podem ser agregadas à discussão da Construção Simbólica. Se um dos entes do Pensamento Computacional é a linguagem, essa tem sua notação e, como tal, apresenta uma utilidade que deve ser construída.

Claro que, na mesma linha de pensamento apresentada por Olson, Campebell (1994), Harel e Kaput (2002), a notação não deve ser o elemento principal, mas sim, uma porta de entrada para auxiliar novas formas de pensar.

Dessa maneira, ao tentar estabelecer essa comunicação entre o Pensamento Matemático e o Pensamento Computacional, mais especificamente entre as ideias da representação simbólica e a linguagem, é possível que os estudantes desenvolvam novas habilidades, e, além disso, possam dar significado a um determinado símbolo nas suas diversas representações, mostrando que um mesmo objeto pode ter outros tipos de representação simbólica.

Esse tipo de atividade desperta no estudante a ideia de que a notação pode fazer a substituição do conceito, ou seja, quando o professor ou o estudante utilizar determinada notação, por trás dessa notação, existe todo um conceito, que o discente tem entendimento e sabe como utilizá-lo, contudo, a notação não substitui o conceito.

A partir do momento que o estudante consegue estabelecer a relação entre a notação e o conceito (que está por trás dela), isso pode favorecer o desenvolvimento de outros tipos de representações de um mesmo objeto matemático.

Considera-se a Construção Mental um ponto da teoria bem complexo, pois entende-se que não é possível mensurar questões relacionadas a esse assunto, pois cada um constrói sua própria representação mental.

No entanto, é possível fazer alguma análise a partir do momento em que o estudante (partindo da perspectiva do professor) externaliza o seu pensamento e, sob essa perspectiva, um ente que se considera essencial é a visualização.

Partindo do princípio que o desenvolvimento de uma construção mental está baseado em um sistema de representações, o Pensamento Computacional pode auxiliar, pois se leva em conta a automação dos processos que envolvem a abstração, em que uma de suas características parte da construção de 
representações concretas, ou seja, algo concreto e tangível. Entende-se que isso colabora para que o estudante tenha uma representação mental rica e, além disso, contribui para a interação e observação de padrões.

Assim, o uso do computador, no processo, pode contribuir, pois alguns programas, tais como Geogebra, Scilab, etc, permitem que o estudante tenha, pelo menos, dois tipos de representações, a algébrica e a geométrica. Nesse processo, ocorrem algumas trocas de representações, colaborando para que o estudante comece a ter sua própria Construção Mental.

Deve-se destacar que esse processo de criação é construído com base em representações concretas que são elaboradas pelo docente. Assim, o professor pode preparar atividades instrucionais, utilizando os softwares citados e as ideias de Automação para processos envolvendo Abstração. Assim, é possível que, nesse movimento, o estudante construa sua representação concreta e arquitete sua própria representação mental.

Por exemplo, a ação do professor não deve ser a costumeira (definição, exemplos e exercícios). Nesse tipo de atividade, seria interessante que alguns conceitos fossem evidenciados pelos próprios estudantes durante a prática.

Levando em consideração ambas as definições do conceito de Modelagem, o processo como um todo parte de algo concreto e, além disso, durante a realização, o estudante está partindo de uma situação micro para macro, esperando, assim, que consiga entender o processo de modo geral, mas de forma detalhada.

Ao se discutir a realização da atividade, parte-se de algo concreto e, à medida que se inicia a discussão e a cada etapa, descobre-se algo, que se denomina de micro, mas conforme o debate vai ocorrendo, novos elementos vão se agregando. Assim, nesse movimento, o processo passa por uma situação bem minuciosa e, dessa maneira, o estudante começa a ter não só uma visão global, mas também local sobre o conteúdo.

Por último, podem-se utilizar ainda algumas das características do Pensamento Computacional - a depuração, pois ela permite olhar a atividade como um todo e verificar se existe ou não algum erro e, em caso positivo, fazer a correção para continuar. Nessa perspectiva, ocorrem condições para construir esquemas, resumos, tabelas, etc.

Outro fator que se destaca no desenvolvimento dessa teorização é que a atuação docente em sala de aula pode contribuir para evidenciá-la, ou seja, o Pensamento Matemático-Computacional estabelece a relação docente - aluno e aluno - docente.

Dessa maneira, acredita-se que a forma para se observar o desenvolvimento do Pensamento MatemáticoComputacional se encontra no diálogo que ocorre nas atividades de sala.

\section{CONSIDERAÇÕES FINAIS}

Este artigo teve como objetivo apresentar uma teorização, tendo como base os processos do Pensamento Matemático Avançado apresentados por Dreyfus (2002) e as concepções do Pensamento Computacional.

Assim, o Pensamento Matemático-Computacional apresenta algumas características, com relação à Construção Simbólica: a construção dos símbolos, relação entre o símbolo e o conceito e outros tipos de representação de um conceito (algébrico, geométrico, etc.), sendo essas construídas por meio das ideias de representação simbólica e a linguagem e sua importância para a computação. 
A Construção Mental está baseada em um sistema de representações e, dessa maneira, pode-se construir uma representação concreta e por meio dela fazer interações e observações. Essas ideias surgiram a partir das reflexões dos processos de representação mental e automação que envolvem processos de abstração.

Espera-se que o Pensamento Matemático-Computacional possa contribuir na aprendizagem. No entanto, o fato de ser um assunto novo pode ainda ter outras considerações, sugestões e críticas. Acredita-se que, com este trabalho, outros pesquisadores das diversas áreas do conhecimento possam dar sua contribuição para que esse tema seja consolidado.

Mesmo assim, pode-se dizer que o Pensamento Matemático-Computacional deve ser entendido como uma atividade mental e cultural. Logo, não existe uma verdade absoluta, mas sim diversas formas de pensar; em que vários aspectos estão relacionados ao contexto.

\section{REFERÊNCIAS}

ASTRACHAN, O. Computational Thinking as the Automation of Abstractions. In: Committee for the Workshop on Computational Thinking; National Research Council. Report of a Workshop on the Scope and Nature of Computational Thinking: National Academies Press, Washington, DC, 2010, p. 14.

BOHR, N. Computational Thinking as the Automation of Abstractions. In: Committee for the Workshop on Computational Thinking; National Research Council. Report of a Workshop on the Scope and Nature of Computational Thinking: National Academies Press, Washington, DC, 2010, p. 13.

COMMITTEE FOR THE WORSHOP ON COMPUTATIONAL THINKING; NATIONAL RESEARCH COUNCIL. Motivation - Why Should Anyone Care About Computational Thinking?. Report of a Workshop on the Scope and Nature of Computational Thinking, Washington, DC, 2010, p. $16-17$.

DREYFUS, T. Advanced Mathematical Thinking. In: TALL, D. Advanced Mathematical Thinking; Kluwer Academic, New York, 2002, p. 25 - 40.

diSESSA A. Computational Thinking as the Automation of Abstractions. In: Committee for the Workshop on Computational Thinking; National Research Council. Report of a Workshop on the Scope and Nature of Computational Thinking: National Academies Press, Washington, DC, 2010, p. 14.

FOX, E. A. Computational Thinking as the Automation of Abstractions. In: Committee for the Workshop on Computational Thinking; National Research Council. Report of a Workshop on the Scope and Nature of Computational Thinking: National Academies Press, Washington, DC, 2010, p. 16.

GOODE, W.J.; HATT, P.K. Métodos em pesquisa social. São Paulo: Nacional, 1979, p. 12.

HAREL, G; KAPUT, J. The Role of Conceptual Entities and their symbols in building Advancede Mathematical Concepts. In: TALL, D. Advanced Mathematical Thinking; Kluwer Academic, New York, 2002, p. 82 - 93.

KAY, A. Computational Thinking as Language and the Importance of Programing. In: Committee for the Workshop on Computational Thinking; National Research Council. Report of a Workshop on the Scope and Nature of Computational Thinking: National Academies Press, Washington, DC, 2010, p. 13 -16. 
KHAN, K. Computational Thinking as the Automation of Abstractions. In: Committee for the Workshop on Computational Thinking; National Research Council. Report of a Workshop on the Scope and Nature of Computational Thinking: National Academies Press, Washington, DC, 2010, p. 16 - 17.

LEE, P. Computational Thinking as the Automation of Abstractions. In: Committee for the Workshop on Computational Thinking; National Research Council. Report of a Workshop on the Scope and Nature of Computational Thinking: National Academies Press, Washington, DC, 2010, p. 16 - 17.

MARTINEAU, S.; SIMARD, D.; GAUTHIER, C. Recherches théoriques et spéculatives: considérations méthodologiques et épistémologiques. Recherches Qualitatives, Montreal, v. 22, n. 3, p. 32, 2001.

OLSON, D. R; CAMPBELL, R. N. Representation and Misrepresentation: On the Beginnings of Symbolization in Young Children. In: TIROSH, D; Implicit and Explicit Knowledge an Educational Approch, Ablex Publishing, New Yourk, 1994, p. 83 - 96. Disponível em: https://www.researchgate.net/publication/893482958. Acesso em: 17 nov. 2016.

PEA, R. Computational Thinking as the Automation of Abstractions. In: Committee for the Workshop on Computational Thinking; National Research Council. Report of a Workshop on the Scope and Nature of Computational Thinking: National Academies Press, Washington, DC, 2010, p. 13.

PLOT. B. Écrire une thèse ou un mémoire en sciences humaines. Paris. Librairie, Honoré Champion Éditeur, 1986.

RESNICK, M. Computational Thinking as Language and the Importance of Programing. . In: Committee for the Workshop on Computational Thinking; National Research Council. Report of a Workshop on the Scope and Nature of Computational Thinking: National Academies Press, Washington, DC, 2010, p. $13-16$.

SCHLANGER, J. L'invention intellectuelle. Paris : Fayard, 1983.

VINNER, S. The Role of Definitions in The Teaching and Learning of Mathematics. In: TALL, D. Advanced Mathematical Thinking; Kluwer Academic, New York, 2002, p. $65-79$.

WIELINSKY, U. Computational Thinking as the Automation of Abstractions. In: Committee for the Workshop on Computational Thinking; National Research Council. Report of a Workshop on the Scope and Nature of Computational Thinking: National Academies Press, Washington, DC, 2010, p. 17.

WING, J.M. Computational Thinking. In: Communications of the ACM. New York: vol 49, $n^{\circ} 3$, march 2006, p $33-35$.

WING, J. M. The Landscape of Computational Thinking. In: Committee for the Workshop on Computational Thinking; National Research Council. Report of a Workshop on the Scope and Nature of Computational Thinking: National Academies Press, Washington, DC, 2010, p. 8 - 10.

WING, J. M. Computational Thinking: What and Why. Disponível em: https://www.cs.cmu.edu/ CompThink/ resources/TheLinkWing.pdf. Acesso em: 10 out. 2017.

WOLZ, U. Computational Thinking as the Automation of Abstractions. In: Committee for the Workshop on Computational Thinking; National Research Council. Report of a Workshop on the Scope and Nature of Computational Thinking: National Academies Press, Washington, DC, 2010, p. 13. 